\title{
Concurrent Mucosal Melanoma and Angiofibroma of the Nose
}

\author{
Jae Hyung Hwang ${ }^{1} \cdot$ Jin Bu Ha ${ }^{1}$ Junguee $\mathrm{Lee}^{2} \cdot$ Joohyung Lee $^{1}$ \\ Departments of ${ }^{l}$ Otorhinolaryngology-Head and Neck Surgery, ${ }^{2}$ Pathology, The Catholic University of Korea College of Medicine, Seoul, Korea
}

Malignant melanoma rarely develops in the paranasal sinuses, and generally has a poor prognosis. However, mucosal melanoma can masquerade both clinically and histopathologically as a benign lesion, rendering accurate early diagnosis difficult. On the other hand, angiofibroma, a benign tumor, is more easily diagnosed than a mucosal melanoma, because the former exhibits specific histopathological features. No cases of concurrent angiofibroma and mucosal melanoma have been reported to date. We describe such a case below.

Keywords. Melanoma; Angiofibroma; Nasal Cavity

\section{INTRODUCTION}

Mucosal melanoma is a malignant tumor with a poor prognosis. Histiopathologically, mucosal melanoma often presents with epidermal hyperplasia, vascularization, hemorrhage, and/or fibrosis, while melanin may be present or absent. Furthermore, clinical symptoms are nonspecific. Therefore, mucosal melanoma can masquerade both clinically and histopathologically as a benign lesion. Hence, it is difficult to reach an early diagnosis of musosal melanoma.

On the other hand, angiofibroma, a benign tumor, is more readily diagnosed than mucosal melanoma because the histopathological findings are specific, including many extensively dilated vascular channels. To the best of our knowledge, there have been no reported cases of concurrent angiofibroma and mucosal melanoma. We report a case of mucosal melanoma of the nasal cavity with concurrent angiofibroma.

\footnotetext{
- Received August 26, 2015

Revised November 24,2015

Accepted December 8, 2015

- Corresponding author: Joohyung Lee

Department of Otorhinolaryngology-Head and Neck Surgery, Daejeon St. Mary's Hospital,The Catholic University of Korea College of Medicine, 64 Daeheung-ro, Jung-gu, Daejeon 34943, Korea

Tel: +82-42-220-9580, Fax: +82-42-221-9580

E-mail: fpsljh@gmail.com
}

\section{CASE REPORT}

A 59-year-old female was admitted to the ear, nose, and throat clinic of Daejeon St. Mary's Hospital in December 2013 complaining of nasal stuffiness and intermittent epistaxis from the right nasal cavity over the past year. She had no systemic disease and no relevant medical or family history. On physical examination, rhinoscopy revealed a polypoid mass in the right nasal cavity, which bled when lightly touched (Fig. 1). A computed tomography (CT) scan revealed a dense soft tissue mass in the right paranasal sinuses and the right nasal cavity (Fig. 2). Magnetic resonance imaging (MRI) revealed an enhanced right antral mass $(3.2 \mathrm{~cm} \times 2.5 \mathrm{~cm} \times 2.2 \mathrm{~cm}$ in size) with a widened maxillary ostium that extended to the right nasal cavity (Fig. 3). The patient underwent endoscopic sinus surgery; however, the mass bled profusely and only a portion was removed for biopsy. Surgery was terminated after the bleeding was controlled. Because of such bleeding tendency, angiography accompanied by tumor embolization was performed prior to reoperation (Fig. 4). The pathologic report of specimen from primary uncompleted operation pointed to a case of the angiofibroma, which was consistent with the patient's clinical features. And we considered right endoscopic medial maxillectomy at revision operation.

In the revision operation the tumor mass was found to be dark greenish and yellowish fragile, filling middle meatus and extending to the nasal cavity. Bleeding was not severe due to the embolization. Its medial extent was to the nasal septum, lat-

Copyright (C) 2016 by Korean Society of Otorhinolaryngology-Head and Neck Surgery.

This is an open-access article distributed under the terms of the Creative Commons Attribution Non-Commercial License (http://creativecommons.org/licenses/by-nc/4.0)

which permits unrestricted non-commercial use, distribution, and reproduction in any medium, provided the original work is properly cited. 

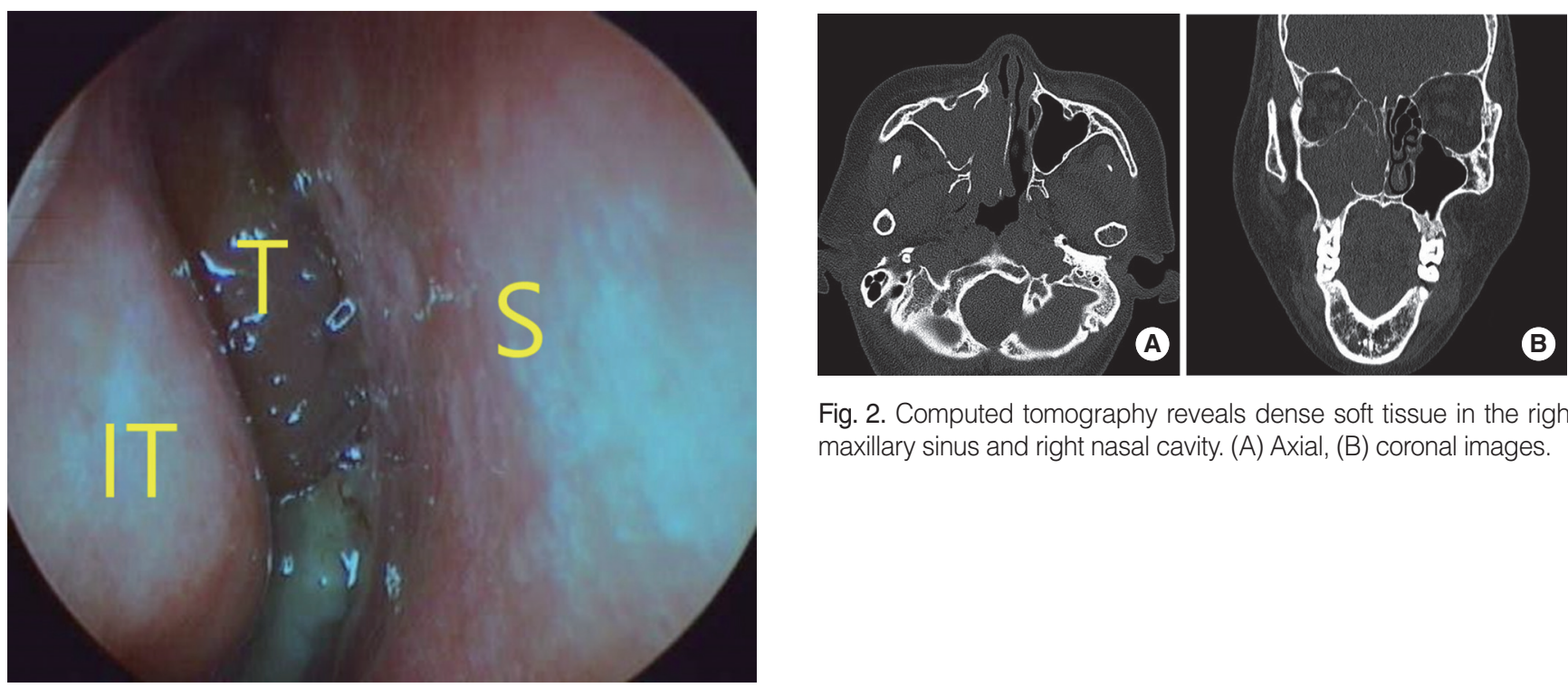

Fig. 2. Computed tomography reveals dense soft tissue in the right maxillary sinus and right nasal cavity. (A) Axial, (B) coronal images.

Fig. 1. Preoperative endoscopic findings in the right nasal cavity. S, septum; IT, inferior turbinate; T, tumor.
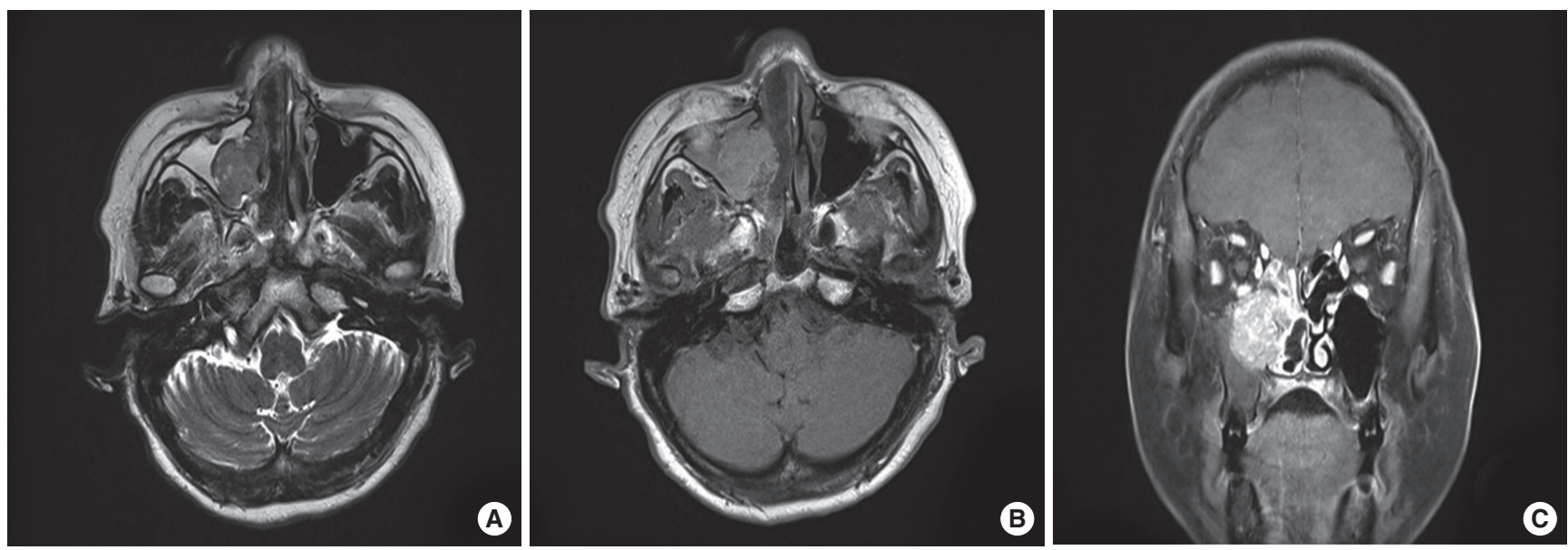

Fig. 3. Paranasal sinus magnetic resonance imaging findings. An enhanced right antral mass with a widened maxillary ostium is evident; it extends into the right nasal cavity axial T2-weighted (A), axial T1-weighted (B), and coronal gadolinium-enhanced T1-weighted (C) images.

eral to the lateral wall of maxillary sinus, superior to middle of the ethmoid sinus, inferior to the inferior maxillary wall, anterior to the anterior maxillary wall, and posterior to the nasopharynx. The origin of the tumor was deduced to be the lateral wall of the nasal cavity near the middle meatus. Furthermore, a large part of the tumor was not adherent to the adjacent sinus wall

\section{H I G G H L I G G H T S}

- This case suggested possibility of coexistence of mucosal melanoma and hemangioma in the nasal cavity neoplasm.

- Malignant condition should be considered in easily bleeding masses in the nasal cavity as of its differential diagonosis. except around the middle meatus.

The final pathology was confirmed to be mucosal melanoma with concurrent angiofibroma. Excised masses were composed of angiofibroma components, which contained thin walled blood vessels and fibrous stroma and mucosal melanoma components, which contained hyperpigmentation in subepithelium on pathologic examination (Fig. 5A-C).

The patient was discharged with no complications, and we scheduled positron emission tomography (PET) CT and consultations with medical and radiation oncologists. PET CT yielded no evidence of a remnant tumor or distant metastasis. The patient underwent adjuvant radiation therapy with immunotherapy (interferon- $\alpha$ ) for 1 year, and intensity-modulated radiotherapy of the right paranasal sinus (5,000 cGy/25 fractions). 


\section{DISCUSSION}

Melanomas usually arise from melanocytes, which are in turn derived from the neuroectoderm of the basal layers of the skin. They develop more rarely in mucosal membranes [1]. Melanomas of the nasal cavity or paranasal sinuses are rarer than cutaneous melanomas; the former tumors constitute $3.6 \%-4 \%$ of all nasal cancers. Mucosal melanomas in the head-and-neck region account for half of all mucosal melanomas. Apart from the head-and-neck region, they can arise in the genital organs of females or the anorectal and urinary tracts, and are far more ag-
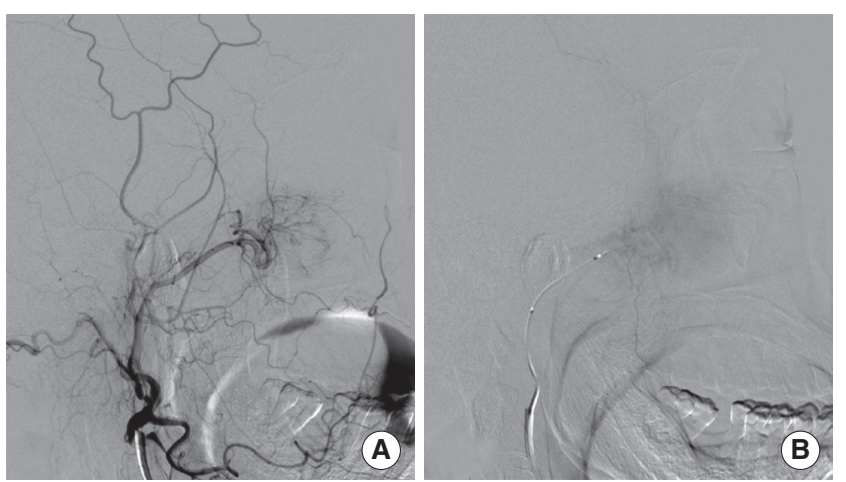

Fig. 4. Angiography with tumor embolization. The tumor was supplied by nasal branch of right sphenopalatine artery. (A) Before embolization, (B) after embolization. gressive with poorer prognoses than cutaneous melanomas [2]. The 5-year survival rate is $10 \%-40 \%$ and the median survival time is $21-24$ months [3]. Such poor prognoses are attributable to both local recurrence and metastasis [4]. Furthermore, primary mucosal melanomas are often misdiagnosed due to their anatomical locations and the lack of discernible signs and symptoms. Epistaxis and nasal obstruction are common symptoms in both mucosal melanoma and nasal benign tumors. CT is used to evaluate the primary tumor and cervical lymph nodes, whereas MRI is employed to explore the extent of sinonasal tumors, particularly those that may involve the skull base and/or exhibit neurotropic spread [5]. Also, mucosal melanoma may either contain or lack melanin, while exhibiting microscopic findings such as epidermal hyperplasia, vascularization, hemorrhage, and/or fibrosis [6]. Immunohistochemical staining may be useful to distinguish melanomas from other malignancies. Melanomas are likely to be positive for HMB-45, Melan-A, S-100 protein, Mart-1, and tyrosinase [2].

The primary treatment modality is surgical resection with wide margins since incomplete resection is associated with poor survival rate [7]. In addition, postoperative radiotherapy should be considered for most cases because of the high risk of local recurrence even after apparently complete resection. Even patients with relatively localized tumors should be considered for such therapy [5]. Chemotherapy is prescribed when a distant metastasis and/or recurrence is/are evident; immunotherapy
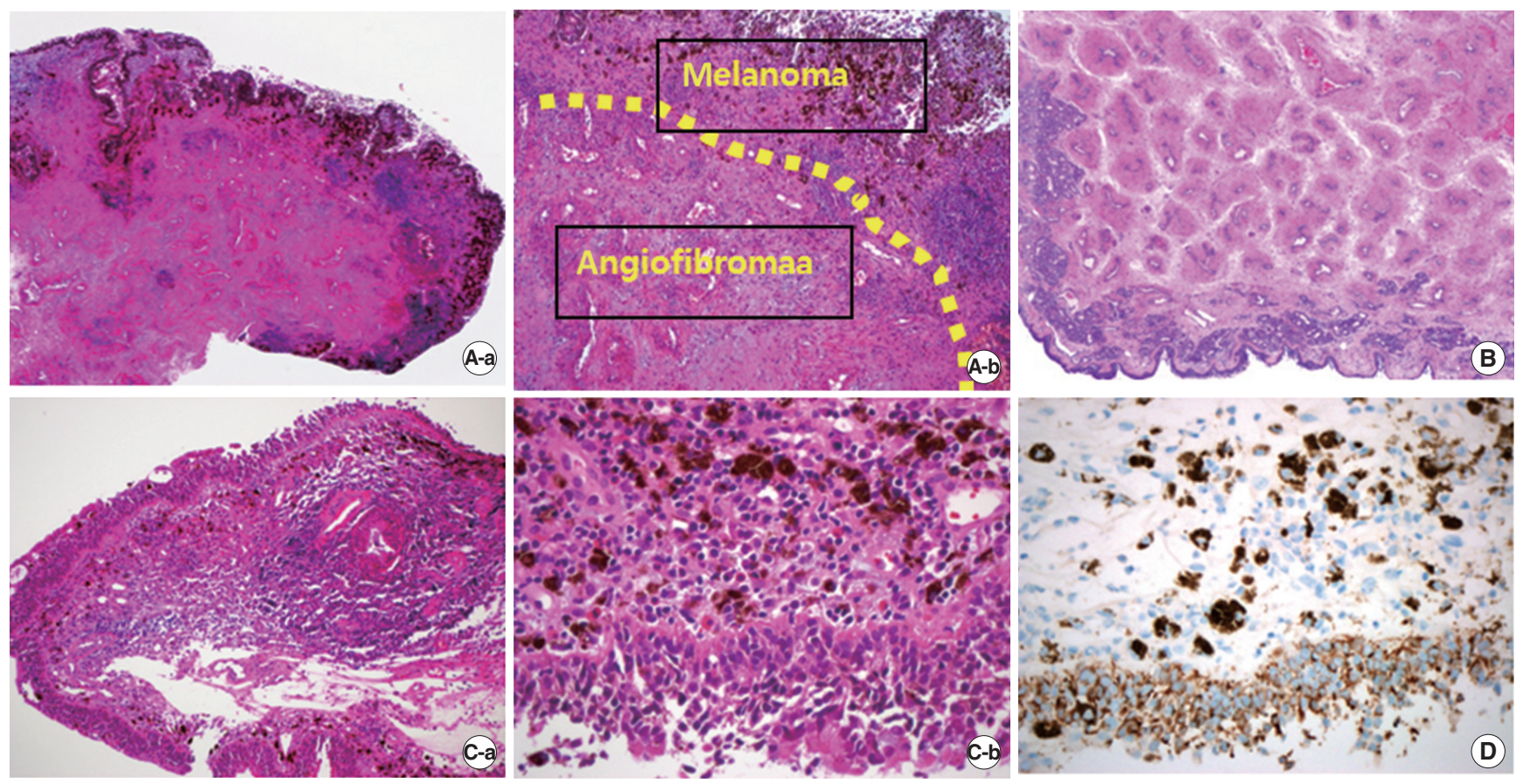

Fig. 5. (A) Mucosal melanoma with an angiofibroma. Mucosal hyperpigmentation in subepithelium showing admixture of thin walled blood vessels and fibrous stroma (H\&E: $a, \times 40 ; b, \times 100)$. (B) The angiofibroma lesion of the specimen. Nasal mucosa with fibroblastic stroma and thin walled blood vessels (H\&E, ×40). (C) Mucosal melanoma with intracytoplasmic melanic pigment (H\&E: a, $\times 100 ; b, \times 400)$. (D) Tumor cells positive for Melan-A $(\times 40)$. 
(Bacillus Calmette-Guérin or interferon) remains unreliable.

This is the first English-language report to describe a concurrent mucosal melanoma and angiofibroma. Our patient tended to bleed easily, a clinical sign of an angiofibroma. Moreover, the biopsy sample included angiofibroma tissue, leading the pathologist to diagnose melanocytes as hemosiderin-laden macrophages (Fig. 5A, B). The mucosal melanoma was ultimately diagnosed via Melan-A staining (Fig. 5D).

In conclusion, mucosal malignant melanomas are very rare, often presenting with atypical symptoms and masquerading both clinically and histologically as benign lesions. When a mucosal melanoma and a benign sinusoidal tumor, such as angiofibroma occur together, accurate diagnosis is difficult. Even if a benign sinusoidal tumor is suspected, careful examination is required. Immunohistochemical staining is required if there is even the slightest suspicion of a mucosal melanoma.

\section{CONFLICT OF INTEREST}

No potential conflict of interest relevant to this article was reported.

\section{REFERENCES}

1. Medhi P, Biswas M, Das D, Amed S. Cytodiagnosis of mucosal malignant melanoma of nasal cavity: a case report with review of literature. J Cytol. 2012 Jul;29(3):208-10.

2. Mihajlovic M, Vlajkovic S, Jovanovic P, Stefanovic V. Primary mucosal melanomas: a comprehensive review. Int J Clin Exp Pathol. 2012 Oct;5(8):739-53.

3. Mundra RK, Sikdar A. Endoscopic removal of malignant melanoma of the nasal cavity. Indian J Otolaryngol Head Neck Surg. 2005 Oct; 57(4):341-3.

4. Rinaldo A, Shaha AR, Patel SG, Ferlito A. Primary mucosal melanoma of the nasal cavity and paranasal sinuses. Acta Otolaryngol. 2001 Dec;121(8):979-82.

5. Mendenhall WM, Amdur RJ, Hinerman RW, Werning JW, Villaret DB, Mendenhall NP. Head and neck mucosal melanoma. Am J Clin Oncol. 2005 Dec;28(6):626-30.

6. Grant-Kels JM, Bason ET, Grin CM. The misdiagnosis of malignant melanoma. JAm Acad Dermatol. 1999 Apr;40(4):539-48.

7. Clifton N, Harrison L, Bradley PJ, Jones NS. Malignant melanoma of nasal cavity and paranasal sinuses: report of 24 patients and literature review. J Laryngol Otol. 2011 May;125(5):479-85. 